\title{
OUR EXPERIENCES WITH MEASUREMENT OF NEW POTENTIAL BIOMARKERS IN THE DIAGNOSIS OF LATENT FORMS OF MYOCARDIAL ISCHEMIA
}

\author{
David Stejskal $^{\mathrm{a} *}$, Borek Lacnak ${ }^{\mathrm{a}}$, Michal Karpisek ${ }^{\mathrm{b}}$, Milan Kaminek ${ }^{\mathrm{c}}$
}

\author{
a Department of Laboratory Medicine and Internal Department, Sternberk Hospital, Czech Republic \\ $b$ University of Veterinary and Pharmaceutical Sciences, Brno \\ c Department of Nuclear Medicine, Faculty of Medicine and Dentistry, Palacky University Olomouc, Czech Republic \\ e-mail:david.stejskal@nemstbk.cz
}

Received: September 15, 2006; Accepted: November 15, 2006

Key words: Myosin Light Chains-1/Reg-Ia/-Clusterin/Latent Myocardial Ischemia

\begin{abstract}
Background: The need for a laboratory marker of myocardial ischemia has been alluded to for at least the last decade.

Aim: The aim of this study was to evaluate the diagnostic importance of the myosin light chain-1 (MLC-1), clusterin and Reg-I $\alpha$ in patients with suspected myocardial ischemia.

Methods: A group of 176 at high-risk for myocardial ischemia subjects was evaluated and divided into two subgroups using myocardial SPECT (Single Photon Emission Computed Tomography) - individuals with and without signs of myocardial ischemia. Laboratory markers in venous blood were repeatedly examined in all subjects: a) immediately prior to SPECT: C-reactive protein, Haemoglobin, Hematocrite, Lactate, MLC-1, Clusterin, Reg-I $\alpha$ b) at subjective maximum: Hb, Htc, lactate, MLC-1, Clusterin, Reg-I $\alpha$ c) 30 min after stress levels reached their peak: MLC-1, Clusterin, Reg-I $\alpha$ and d) 60 min after peak stress levels: MLC-1, Clusterin, Reg-I $\alpha$.

Results: Patients were divided into subgroups according to their positive and negative SPECT results (positive: $\mathrm{n}=37$; negative: $\mathrm{n}=139$ ). MLC-1 values were different for all 4 blood collections. An increase in MLC-1 > $2.2 \mathrm{mg} / 1$ showed $64 \%$ sensitivity and $88 \%$ specificity for the diagnosed presence of myocardial ischemia (AUC 0.81; LR+ 5.9; PPV+ $68 \%$ and NPV- $87 \%$ ). There was no significant difference between the groups in terms of Clusterin and Reg-I $\alpha$ for any of the sampling periods.
\end{abstract}

Conclusions: High diagnostic efficacy of detectable MLC-1 was shown for the diagnosis of latent myocardial ischemia. Measurement of serum Clusterin or Reg-I $\alpha$ did not sufficient for the diagnosis of latent myocardial ischemia.

\section{INTRODUCTION}

In the last few years several papers have reported on the need for a new sensitive and in particular early test for myocardial ischemia which would allow an early and efficient therapeutic intervention. The search is based on the commonly known disadvantages with the routinely used laboratory markers of myocardial events (cardiac troponins - detect only myocardial necrosis) and performance of stress testing (e.g. exercise stress test, myocardial Single Photon Emission Computed Tomography - SPECT), which are time-consuming and rather expensive ${ }^{1-5}$.

A promising laboratory markers can be serum clusterin, Reg-I $\alpha$ and myosin light chains-1 (MLC-1). Only a few papers have described the good diagnostic efficacy of these markers; Clusterin, Reg-I $\alpha$ and MLC-1 appears to have a high diagnostic efficacy for the assessment of myocardial damage. There is no valid information about MLC-1, Clusterin or Reg-I $\alpha$ assessment in the diagnosis of latent or transient forms of myocardial ischemia.

The aim of the present paper was to confirm a possible diagnostic utility for the above-mentioned markers in the diagnosis of latent myocardial ischemia.

\section{METHODS}

\section{Patients}

A group of 176 subjects was tested ( 84 men and 92 women). Tested individuals were asymptomatic patients with a high risk of latent myocardial ischemia (individuals with numerous risk factors and risk according to the Framingham algorithm over $20 \%$ / 10 years or the Cardio Score algorithm over $5 \%$ / 10 years) or individuals with a history of asymptomatic forms of coronary artery disease (CAD). None of the tested subjects had signs of heart failure, acute coronary syndrome, and follow up for nephropathy, diabetes mellitus treated with insulin or any inflammatory disease.

All examined subjects gave their informed consent according to the Helsinki Declaration and entered the study.

\section{Myocardial SPECT}

Stress tests: Physical stress was performed on a bicycle ergometer; a radiopharmacum was applied after exceeding $85 \%$ of maximal aerobic capacity or when the exercise was limited by marked angina pectoris, ischemic 
changes on the ECG, severe arrhythmia, reduced blood pressure $^{1-2}$.

Gated SPECT: Gated SPECT was attained $15 \mathrm{~min}$ after application of $740 \mathrm{MBq}{ }^{99 \mathrm{~m}} \mathrm{Tc}-\mathrm{MIBI}$ at maximum stress. In the case of perfusion heterogeneity, resting examination was made another day (gated SPECT 1 hour after application of ${ }^{99 \mathrm{~m}}$ Tc-MIBI). 8-frame gating was used to collect data on a double detector scintillation camera Siemens e.cam (Siemens Medical Solutions, Erlangen, Germany) equipped with parallel collimators of LEHR type, rotation angle of $180^{\circ}$, total 64 projections in a matrix $64 \times 64$ from the a anterior oblique projection of $45^{\circ}$ into the a posterior oblique projection of $45^{\circ}$.

Evaluation of SPECT findings: Tomographic sections of myocardial perfusions were evaluated visually and classified as normal findings and stress induced perfusion defects. Quantitative analysis of perfusion was made on polar maps. Summed stress score (SSS) and summed difference score (SDS) of myocardial perfusion, transient ischemic dilatation (TID), area of left ventricle defect, post stress, and resting LVEF were obtained by means of an automatic quantitative program 4D-MSPECT (University of Michigan, Ann Arbor, USA).

The results of SPECT were considered as standard for the diagnosis of $\mathrm{CAD}^{3-5}$.

\section{Definition of latent forms of myocardial ischemia}

Latent forms of myocardial ischemia were defined as SPECT positivity ( SSS > 3) with negative ECG findings in CAD asymptomatic individuals for use in this paper ${ }^{3-5}$.

\section{Laboratory examination and blood collection}

The examination of biological material was made on venous serum samples, citrate plasma and EDTA blood. All parameters except MLC-1, clusterin and Reg-I $\alpha$ were determined within 120 min after collection.

In all patients, the following laboratory examination in venous blood was made:

a) immediately before SPECT: C-reactive protein (Advia 1650, immunoturbidimetry, serum), Haemoglobin ( $\mathrm{Hb}$ ), Hematocrite (Htc) (MaxM Coulter, EDTA blood), lactate (Advia 1650, citrate plasma), MLC-1, Clusterin, Reg-I $\alpha$ (ELISA line Marc+Max, serum), cardiac Troponine I (Immulite Turbo, LEIA, serum)

b) at subjective maximum within SPECT: Hb, Htc, lactate, MLC-1, clusterin, Reg-I $\alpha$

c) 30 min after stress levels reached their peak: MLC-1, Clusterin, Reg-I $\alpha$

d) $60 \mathrm{~min}$ after stress levels reached their peak: MLC-1, Clusterin, Reg-I $\alpha$

To evaluate the dynamics of MLC-1, Clusterin and Reg-I $\alpha$, the whole group was divided into subgroups according to the results of SPECT (persons with signs of myocardial ischemia on SPECT vs. individuals without signs of myocardial ischemia). Division into the abovementioned subgroups was also used for plotting the ROC curve for diagnostic efficacy of MLC-1, Clusterin and Reg$\mathrm{I} \alpha$ with respect to the presence of myocardial ischemia during stress. The group of patients with SPECT positive results ( $\mathrm{SSS}>3$ ) was divided into patients with permanent or reversible form of myocardial ischemia ${ }^{3,4}$.

\section{Laboratory determination of MLC-1, Clusterin and Reg-Ia}

Collection for MLC-1, Clusterin and Reg-I $\alpha$ was made from peripheral venous blood; determination was performed from the serum. After collection, each sample was kept at room temperature for 60 minutes. Then centrifugation was performed ( 10 minutes, $1100 \mathrm{~g}$ at $4{ }^{\circ} \mathrm{C}$ ) and the samples were frozen at $-80{ }^{\circ} \mathrm{C}$.

MLC-1, clusterin and Reg-I $\alpha$ were determined within 3 months after material collection and after adaptation to room temperature $\left(25^{\circ} \mathrm{C}\right)$. All samples were examined in duplicate.

The ventricular form of MLC-1 (vMLC-1) was determined by the ELISA method (Biovendor Inc.) in sandwich arrangement. A combination of two murine monoclonal antibodies was used for determination. Basic analytical characteristics of the method: $\mathrm{CV}$ in a series $<5 \%, \mathrm{CV}$ between series $<8 \%$ in calibration spectrum /width/, functional sensitivity $0.5 \mathrm{mg} / 1$. Analytical specificity: $10 \%$ cross reactivity against MLC-2 and $20 \%$ against MLC skeletal muscles (enclosed leaflet of Biovendor Inc.).

Reg-I $\alpha$ was determined by the ELISA method (Biovendor Inc.) in sandwich arrangement. Basic analytical characteristics of the method: $\mathrm{CV}$ in a series $<4 \%$, CV between series $<9 \%$ in calibration spectrum /width/, functional sensitivity $5 \mu \mathrm{g} / 1$.

Serum Clusterin was determined by the ELISA method (Biovendor Czech Republic, two anti-human Mouse monoclonal antibody, human serum based calibrator) in sandwich arrangement. Basic analytical characteristics of the method: $\mathrm{CV}$ in a series $<6 \%$, CV between series < $9 \%$ in calibration spectrum /width/, functional sensitivity $5000 \mathrm{kU} / 1$.

\section{Statistical data processing}

The data were processed by means of the Medcalc software. Associated constants are expressed as mean \pm standard deviation and median, unless indicated otherwise. The levels of MLC-1 in the subgroups were compared using variance analysis (ANOVA, Kruskal-Wallis according to distribution type) and by means of ROC analysis. The concentration of MLC-1, clusterin and RegI $\alpha$ as well as other quantities were mutually correlated using a Spearman correlation coefficient. Category data were compared by using $\chi^{2}$ test. Probability value $p<0.05$ was considered as statistically significant. Normality was evaluated by the $\chi^{2}$ test.

\section{RESULTS}

37 individuals were SPECT positive (27\%), 139 subjects $(63 \%)$ had no SPECT signs of myocardial ischemia.

After division into two subgroups according to SPECT 


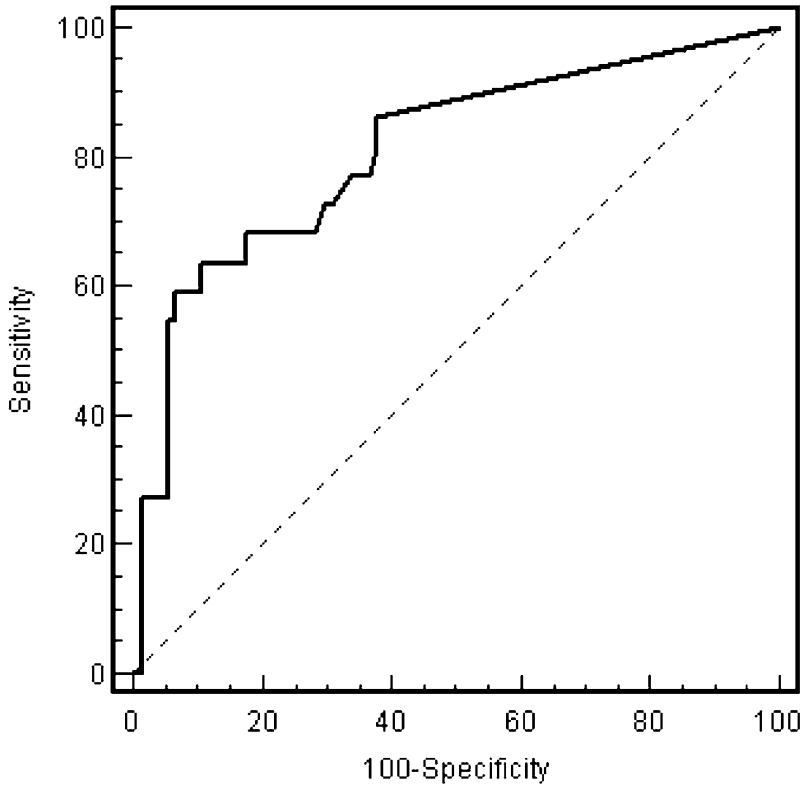

Fig. 1. Receive operating curve (ROC) for MLC-1 prior SPECT with respect to myocardial ischemia signs at SPECT

for presence of myocardial ischemia, the two subgroups differed significantly only in MLC-1 concentrations in all 4 collections (medians in $\mathrm{mg} / \mathrm{l} ; 3.1$ vs. 0.5 in collection 1 ; 3.1 vs. 1.3 in collection $2 ; 2.9$ vs. 0.5 in collection $3 ; 3.4$ vs. 0.5 in collection 4$)$. The age of subjects, as well as lactate, CRP, Reg-I $\alpha$, Clusterin or energetic output in endurance testing did not differ significantly. Individuals with negative SPECT results had significantly higher values of maximum heart rates and stress LVEF. As the SSS, SDS, area of left ventricular defect and TID are comprised in the evaluation criteria for SPECT, a significant difference is not seen here (Tab. 1). MLC-1 values did not differ by gender $(\mathrm{p}=0.81)$.

Individuals with reversible defects of myocardial perfusion had higher basal values of MLC-1 than individuals with a fixed defect (MLC-1 values did not differ in individuals with a fixed defect of myocardial perfusion and subjects without myocardial ischemia (Tab. 2). Examination of the correlation showed a positive significant relationship between MLC-1 prior SPECT and the value of SSS $(c=0.27 ; p=0.01), \operatorname{SDS}(c=0.26 ; p=0.01)$, area of left ventricle defect $(c=0.27 ; p=0.01)$. A negative correlation between MLC-1 concentrations prior to stress and maximum heart rate $(\mathrm{c}=-0.23 ; \mathrm{p}=0.01)$ was detected. Very close correlations were found between MLC-1 during stress $(0.92-0.97 ; \mathrm{p}<0.01)$. No other significant correlations were observed between MLC-1 under basal conditions or during stress and any other measured parameters. Especially interesting is the finding in the values of serum creatinin, LVEF, stressEF a restEF, which could be expected on the basis of preliminary results obtained in other studies. With respect to the insignificant differences in MLC-1 during repeated collection, the diagnostic efficacy of MLC-1 was tested under basal conditions for

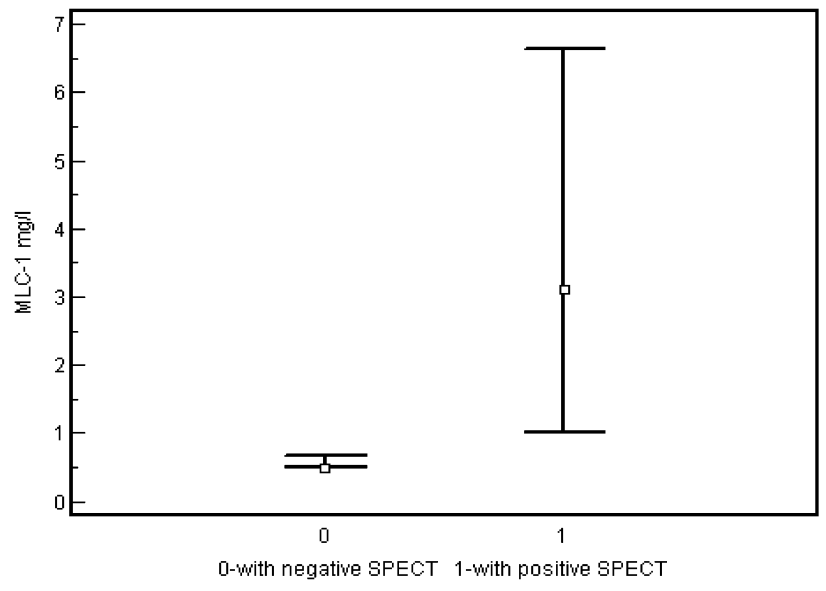

Fig. 2. Comparison of MLC-1 values prior SPECT (demonstrated median $+95 \% \mathrm{CI}$ ) in patients according to SPECT signs of myocardial ischemia

the diagnosis of myocardial ischemia detected by SPECT. Figures 1 and 2 indicate $64 \%$ sensitivity and $88 \%$ specificity for the diagnosis of the presence of myocardial ischemia for MLC-1 over $2.2 \mathrm{mg} / 1$ (AUC 0.81; $95 \% \mathrm{CI}$ $0.72-0.88$; LR+ 5.9; PPV+ in our group was $68 \%$ and NPV $-87 \%$ ).

\section{DISCUSSION}

Diagnosis of myocardial ischemia is based on a combination of many tests. No gold standard for the profile of myocardial ischemia diagnosis currently exists.

SPECT appears to be possible diagnostic method for myocardial ischemia estimating. Recent papers have acknowledged the diagnostic importance of SPECT for risk stratification and therapy planning ${ }^{1-5}$.

Patients with normal stress myocardial perfusion have a good prognosis in cardiovascular complication occurrence ${ }^{3-5}$.

Individuals with mild myocardial ischemia (mild abnormality of myocardial perfusion without post ischemic myocardial stunning, SSS values between 4-8; TID < 1.17; no decline in stress LVEF) have a relatively favourable prognosis and can be treated conservatively. Invasive intervention has to be executed at SSS values $>9$ as well as in situations with SSS between 4-8 and signs of myocardial stunning are present at the same time (TID > 1.17 or declension of stress LVEF $)^{1-5}$. For all SPECT is very efficacious on myocardial ischemia diagnostics; this is a time-consuming, rather sought after. The examination of some proteins with different expression through myocardial injury is one of the possibilities for myocardial ischemia testing. 
Table 1. Measured parameters, two subgroups according to signs of myocardial ischemia using SPECT

(Single Photon Emission Computed Tomography).

\begin{tabular}{|c|c|c|c|c|c|c|c|c|c|}
\hline \multirow[t]{2}{*}{ Parameter (units) } & \multicolumn{4}{|c|}{$\begin{array}{l}\text { Myocardial ischemia } \\
\text { YES, } n=37\end{array}$} & \multicolumn{4}{|c|}{$\begin{array}{c}\text { Myocardial ischemia } \\
\text { NO, } n=139\end{array}$} & \multirow[t]{2}{*}{ Differences } \\
\hline & $\mathrm{X}$ & Median & SD & $\begin{array}{l}\text { Nor- } \\
\text { mality }\end{array}$ & $X$ & Median & SD & $\begin{array}{l}\text { Nor- } \\
\text { mality }\end{array}$ & \\
\hline MLC1-1 (mg/l) & 3.8 & 3.1 & 3.1 & No & 1.6 & 0.5 & 4.9 & No & $* \mathrm{p}<0.0001$ \\
\hline MLC1-2 (mg/1) & 4.1 & 3.1 & 3.2 & No & 1.8 & 1.1 & 3.9 & No & $* \mathrm{p}<0.0001$ \\
\hline MLC1-3 (mg/1) & 3.9 & 2.9 & 3.0 & No & 2.0 & 0.5 & 5.4 & No & $* \mathrm{p}=0.0003$ \\
\hline MLC1-4 (mg/l) & 4.2 & 3.4 & 3.3 & No & 1.8 & 0.5 & 4.9 & No & $* \mathrm{p}=0.0002$ \\
\hline Clusterin-1 (kU/1) & 157069.0 & 151298.0 & 21287.0 & Yes & 171764.0 & 168987.0 & 24872.0 & Yes & $p=0.13$ \\
\hline Clusterin-2 (kU/1) & 182392.0 & 171311.0 & 24369.0 & Yes & 181882.0 & 179126.0 & 21239.0 & Yes & $p=0.97$ \\
\hline Clusterin-3 (kU/1) & 161564.0 & 163218.0 & 29850.0 & Yes & 152347.0 & 154784.0 & 28987.0 & Yes & $p=0.29$ \\
\hline Clusterin-4 (kU/l) & 164429.0 & 165154.0 & 32254.0 & Yes & 157777.0 & 159137.0 & 22987.0 & Yes & $p=0.59$ \\
\hline Reg-I alpha -1 (ug/l) & 589.9 & 572.2 & 298.9 & No & 700.8 & 552.3 & 751.6 & No & $p=0.59$ \\
\hline Reg-I alpha - 2 (ug/l) & 605.7 & 553.7 & 211.6 & No & 687.4 & 590.1 & 511.6 & No & $p=0.47$ \\
\hline Reg-I alpha - 3 (ug/l) & 511.4 & 581.1 & 305.2 & No & 651.1 & 549.5 & 487.0 & No & $p=0.69$ \\
\hline Reg-I alpha - 4 (ug/l) & 604.8 & 569.4 & 297.4 & No & 697.4 & 542.8 & 397.4 & No & $\mathrm{p}=0.81$ \\
\hline $\mathrm{CRP}(\mathrm{mg} / \mathrm{l})$ & 11.9 & 11.2 & 9.4 & No & 10.7 & 9.6 & 6.9 & Yes & $p=0.61$ \\
\hline Hb1 (g/l) & 142.3 & 146.0 & 13.2 & No & 140.0 & 142.0 & 13.3 & Yes & $p=0.44$ \\
\hline $\mathrm{Hb} 2(\mathrm{~g} / \mathrm{l})$ & 149.7 & 154.5 & 15.2 & No & 147.6 & 150.0 & 13.0 & Yes & $p=0.31$ \\
\hline $\operatorname{Htd}(1)$ & 0.43 & 4.0 & 0.04 & Yes & 0.42 & 0.42 & 0.04 & Yes & $p=0.33$ \\
\hline $\operatorname{Htc} 2(1)$ & 0.46 & 0.48 & 0.05 & No & -0.45 & 0.45 & 0.04 & Yes & $p=0.21$ \\
\hline Lactatei $(\mathrm{mmol} / \mathrm{l})$ & 2.0 & 2.0 & 0.6 & Yes & 2.2 & 0.58 & 0.26 & Yes & $p=0.3$ \\
\hline Lactate2 $(\mathrm{mmol} / \mathrm{l})$ & 6.3 & 6.2 & 2.1 & Yes & 6.5 & 6.7 & 2.2 & Yes & $\mathrm{p}=0.46$ \\
\hline StressEF (\%) & 54.0 & 55.5 & 12.7 & Yes & 66.2 & 69.0 & 8.0 & No & ${ }^{*} \mathrm{p}<0.0001$ \\
\hline RestEF (\%) & 54.9 & 57.5 & 11.1 & Yes & 58.7 & 60.0 & 6.7 & Yes & $p=0.18$ \\
\hline SSS (u) & 10.9 & 9.0 & 9.7 & No & 0.24 & 0.0 & 2.3 & No & ${ }^{*} \mathrm{p}<0.0001$ \\
\hline SDS (u) & 5.2 & 3.0 & 0.95 & No & 0.06 & 0.0 & 0.6 & No & ${ }^{*} \mathrm{p}<0.0001$ \\
\hline Area of LV defect (\%) & 21.3 & 18.5 & 0.83 & No & 0.37 & 0.0 & 8.3 & No & ${ }^{*} \mathrm{p}<0.0001$ \\
\hline TID (u) & 1.18 & 1.16 & 0.21 & Yes & 0.97 & 1.0 & 0.23 & No & ${ }^{*} \mathrm{p}=0.0009$ \\
\hline Heart Rate max (bit/min) & 131.0 & 136.5 & 25.8 & Yes & 148.7 & 154.0 & 21.9 & Yes & ${ }^{*} \mathrm{p}=0.0001$ \\
\hline Output (w/kg) & 1.48 & 1.5 & 0.4 & Yes & 1.5 & 1.5 & 0.48 & Yes & $p=0.73$ \\
\hline Age (years) & 65.4 & 67.0 & 9.0 & Yes & 61.8 & 63.0 & 10.6 & Yes & $\mathrm{p}=0.09$ \\
\hline Sex & \multicolumn{2}{|c|}{28 men, 9 women } & & & \multicolumn{2}{|c|}{56 men, 83 women } & & & $p=0.09$ \\
\hline
\end{tabular}

$\mathrm{X}$ - mean, SD - standard deviation, Differences * - statistically significant differences

$\mathrm{Hb}$ - haemoglobin, Parameter X 1-4 - samples prior SPECT, at subjective maximum, $30 \mathrm{~min}$ after the stress, $60 \mathrm{~m}$ in after the stress; Htc - hematocrit, SSS - summed stress score; SDS - summed dilatation score; StressEF - post stress LVEF; RestEF - resting LVEF; TID - transient ischemic dilatation;

Myosin is a contractile protein composed of two heavy chains (MW $200 \mathrm{kDa}$ ) and two light chains. The light chains are termed MLC-1 (25 kDa) and MLC-2 (19 kDa). In the organism there are several types of MLC (e.g. MLC of skeletal muscles - sMLC, MLC of ventricles - vMLC, MLC of atria - aMLC). The damaged integrity of cardiomyocytes (e.g. myocardial necrosis) leads to the liberation of vMLC-1 from the myocardium into circulation. Increased MLC may be found as early as 3-6 hours after the event and maximal values are reached during day 1-5 after the onset of necrosis (heavy chains occur in circulation on day 2-10 after the onset of necrosis and their maximal values are reached on day 5-6). An enhanced MLC in circulation persists for one week. As MLC is excreted by the kidneys, subjects with damaged kidney function show higher values of MLC and the dynamics of changes are impaired after myocardial necrosis. MLC is considered as marker of myocardial necrosis by displaying sufficient sensitivity and specificity (MLC-1 occurs mostly in the heart as vMLC-1). Unfortunately, poor quality of the diagnostic kits used has been reported. Although MLC-1 is present mostly in ventricle myocardium, is it not myocardium-specific (occurs also in skeletal muscle $)^{6-8}$. Elevated MLC-1 has also been described in other cases of myocardial involvement, in addition to ischemic necrosis (myocarditis). Several recent papers have reported that elevated MLC-1 confirms the diagnosis and, moreover, its concentrations may allow assessment of necrotic focus extent for the short-term and long-term outcome in patients (even with higher validity than troponin concentration $)^{8-11}$. Recently, information has shown the high diagnostic efficacy of MLC-1 for the assessment of minimal myocardial involvement ${ }^{9}$. The disadvantage of most diagnostic kits is their low myocardium specificity 9 . Several papers described enhanced MLC-1 in stabilized patients with cardiac dysfunction (NYHA III); according 
Table 2. MLC-1 values prior SPECT in individuals with respect to fixed or reversible defect of myocardial perfusion at SPECT.

\begin{tabular}{|c|c|c|c|c|c|c|c|c|c|}
\hline \multirow{5}{*}{$\begin{array}{l}\text { Parameter } \\
\text { MLC1-1 }\end{array}$} & \multicolumn{4}{|c|}{ Reversibile defect present $n=25$} & \multicolumn{4}{|c|}{ Reversibile defect no present $\mathrm{n}=151$} & \multirow[t]{2}{*}{ Difference } \\
\hline & $\mathrm{X}$ & Median & SD & Normality & $\mathrm{X}$ & Median & SD & Normality & \\
\hline & 3.11 & 2.76 & 2.81 & Yes & 1.80 & 0.50 & 4.80 & No & $\mathrm{p}=0.005$ \\
\hline & \multicolumn{4}{|c|}{ Fixed defect present } & \multicolumn{4}{|c|}{ Fixed defect no present } & \\
\hline & 1.90 & 0.85 & 2.57 & Yes & 2.00 & 0.50 & 4.74 & No & $\mathrm{p}=0.4$ \\
\hline
\end{tabular}

$\mathrm{X}$ - mean, SD - standard deviation, Difference - statistically significant difference

to MLC-1 concentration, mortality in these patients could be predicted ${ }^{7,10,11,13}$. One of the reasons for elevated MLC1 in circulation in such patients may be due to degeneration of myofibrils with the subsequent release of their components into circulation, which is often associated with cardiac dysfunction. These structural changes may accelerate the dysfunction of ventricles and enhance the filling pressure. Another reason may be over-expression of the MLC-1 gene, which may be due to excessive stress of cardiomyocytes. This event may cause over-expression of protein in myocardial cells and its release into circulation. The question remains open whether such release is associated with damaged structure of cardiomyocytes, that is, whether a damaged structure is a pre-requisite. An increased mortality in patients with elevated MLC-1 may reflect myocytary stress, impaired integrity of myocytary membrane or damaged structure of cardiomyocytes, which are associated with the release of intracellular proteins into circulation. Striking is the information that this phenomenon is observed in patients considered clinically stabilized. MLC-I is also a substrate for caspase-3, which is activated after triggering the apoptotic cascade. Activated caspase- 3 cleaves vMLC-1, thus disrupting the biologically effective vMLC-1, which is associated with reduced contractility of the myocardium. A direct cleavage of vMLC-1 by caspase-3 may result in reduced myocardial function through alteration of crossed bridging between actin and myosin molecules. Thus, activation of the apoptotic pathway in the heart may lead to contractile dysfunction even before cell death ${ }^{12}$. Our finding may support the hypothesis that the release of MLC-1 into circulation occurs during "excessive" stress of cardiomyocytes. Such stress may be either transient or latent myocardial ischemia. It is almost certain that apoptosis (its relation to MLC-1 mentioned above) plays an important role in pathogenesis of CAD.

In 1984, a novel gene was found, which plays role in various types of tissue regeneration ${ }^{14-15}$. Recently, the regenerating gene (Reg) has been documented to play an important role in the regeneration of myocardium and transcriptional activation of Reg in the heart in response to heart stress has been detected. In damaged human myocardium (e.g. myocardial infarction) Reg-1 gene expression in fine granular pattern in the cytoplasm of cardiomyocytes has been located. Reg protein secretion into the serum after ischemia, and circulating levels of the protein after myocardial infarction were higher than those after aortic constriction. These results demonstrate the $\mathrm{Reg} / \mathrm{Reg}$ receptor system in damaged hearts. In view of emerging evidence of Reg for tissue regeneration in a variety of tissues/organs, it is proposed that the damaged heart may be a target for Reg action and that Reg may protect against acute heart stress ${ }^{16}$. However, our finding do not support the hypothesis that the release of RegI $\alpha$ into circulation occurs during "excessive" short-term stress of cardiomyocytes. Expression and concentration of this protein is different, we think.

Clusterin (Apolippoprotein J; SP-40,40; TRPM-2; SGP-2; pADHC-9; CLJ; T64; GP III; XIP8) is a highly conserved disulfide-linked secreted heterodimeric glycoprotein of $75-80 \mathrm{kDa}$ but truncated forms targeted to nucleus have also been identified. The protein is constitutively secreted by a number of cell types including epithelial and neuronal cells and is a major protein in physiological fluids including plasma, milk, urine cerebrospinal fluid and semen ${ }^{17}$. Due to its wide breath of tissue distribution many diverse physiological functions have been attributed to clusterin including sperm maturation, membrane recycling, lipid transportation, tissue remodelling, complement inhibition and cell-cell or cellsubstratum interactions. Moreover, it was propose, that clusterin functions is as an extra cellular chaperon that stabilizes stressed proteins in a folding-competent state and protein has also been implicated in programmed cell death. Another defining prominent of clusterin is its induction in many severe physiological disturbances states including kidney degenerative diseases, prostate and vesicle carcinogenesis, ovarian cancer and several neurodegenerative conditions ${ }^{18-22}$. Recent study demonstrate, that serum clusterin level increases significantly in diabetic type 2 patients and in patients with developing coronary heart disease, or myocardial infarction. These date raise the possibility that elevated clusterin levels in serum may represent a strong indication of vascular damage ${ }^{23-24}$. Up regulation of clusterin may contribute to the potent antiatherosclerotic effects of shear stress by preventing endothelial activation through the complement cascade. Ischemia may be induces the up regulation of clusterin in ischemically challenged, but viable, cardiomyocytes ${ }^{25-26}$. No valid information about clusterin concentrations at repetitive samples in course of stress excercisse tests or in short delay after acute coronary syndromes were written. However, our findings did not support the hypothesis that the release of clusterin into circulation occurs during 
short-term "excessive" or long-term moderate stress of cardiomyocytes in case of occurrence of latent myocardial ischemia. Causation of these facts could be short-term or weak stress of cardiomyocytes.

It can be concluded that our findings are unique for the high diagnostic efficacy of a single assessment of MLC-1 for diagnosis of latent myocardial ischemia evaluated by SPECT. This finding may be of crucial importance for technical, economic and medical reasons. On the contrary, diagnostic efficacy of an assessment of Reg-I $\alpha$ or clusterin for the diagnosis of latent myocardial ischemia is insufficient. Further research is warranted to confirm findings and improve diagnostics for CAD patients.

\section{REFERENCES}

1. Klocke JF. ACC/AHA/ASNC Guidelines for the Clinical Use of Cardiac Radionuclide Imaging. In: ACC/AHA Practice Guidelines.

2. Hesse B, Tägil K, Cuocolo A. EANM/ESC procedural guidelines for myocardial perfusion imaging in nuclear cardiology. Eur J Nucl Med Mol Imaging 2005; 32:855-97.

3. Kaminek M, Skvarilova M, Ostransky J. Transient postischemic stunning evaluation by gated SPECT has the potential to detect patients with multivessel coronary artery disease: Validation of the 4D-MSPECT software. Eur J Nucl Med 2003; 30:254.

4. Abidov A, Bax JJ, Hayes SW. Transient ischemic dilation ratio of the left ventricle is a significant predictor of future cardiac events in patients with otherwise normal myocardial perfusion. SPECT 2003; 42:1818-5.

5. Hachamovitch R. Clinical value of combined perfusion and function imaging in the diagnosis, prognosis, and management of patients with suspected or known coronary artery disease. In Germano G, Berman DS. Clinical gated cardiac SPECT. Armonk, New York: Futura Publishing Company, Inc; 1999. p. 239-58.

6. Hansen MS, Stanton EB, Gawad Y. Relation of Circulating Cardiac Myosin Light Chain-1 Isoform instable Severe Congestive Heart Failure to Survival and Treatment With Flosequinan. Am J Cardiol 2002; 90:969-3

7. Sawada H, Nagata I, Shoraki K. Serum concentration of MLC-1 and left ventricular shortening fraction of neonates. Yonago Acta Medica 1999; 42:69-8.

8. Hillis GS, Taggart P, Hillis L. Biochemical and clinical predictors of long-term outcomes in patients with non-specific chest pain and non-diagnostic electrocardiograms. Am Heart J 2003; 145:88-4.

9. Hillis GS, Zhao N, Dalsey WC. Utility of cardiac troponin I, creatine kinase-MB mass, myosin light chain 1 , and myoglobin in the early in-hospital triage of "high risk" patients with chest pain. Heart 1999; 82:614-20.

10. Goto T, Takase H, Tariyama. Circulating concentrations of cardiac proteins indicate the severity of congestive heart failure. Heart 2003; 89:1303-7.
11. Hillis GS, Taggart P, Wardlaw D, Hillis L, Zhao N, Dalsey WC, et al. The relative utility of cardiac troponin I, creatine kinaseMBmass, and myosin light chain-1 in the long-term risk stratification of patients with chest pain. Clin Cardiol 2003; 26:147-2.

12. Communal C, Sumandea M, Tombe P. Functional consequenses of caspase activation in cardiac myocytes. PNAS 2002; 99:6252-6.

13. Hansen MS, Stanton EB, Gawad Y. Relation of Circulating Cardiac Myosin Light Chain-1 Isoform instable Severe Congestive Heart Failure to Survival and Treatment With Flosequinan. The Am Journal of Cardiology 2002; 90:969-73.

14. Fukui H, Fujii S, Takeda J, Kayahara T, Sekikawa A, Nanakin A, et al.Expression of reg I alpha protein in human gastric cancers. Digestion 2004; 69:177-84.

15. Sanchez D, Gmyr V, Kerr-Conte J, Kloppel G, Zenilman ME, GuyCrotte $\mathrm{O}$, et al: Implication of Reg I in human pancreatic duct-like cells in vivo in the pathological pancreas and in vitro during exocrine dedifferentiation. Pancreas 2004; 29:14-1.

16. Kiji T, Dohi Y, Takasawa S, Okamoto H, Nonomura A, Taniguchi $\mathrm{S}$. Activation of regenerating gene Reg in rat and human hearts in response to acute stress. Am J Physiol Heart Circ Physiol 2005; 289:277-84.

17. Silkensen J. Clusterin: physiologic and pathophysiologic considerations. Int J Biochem Cell Biol 1995; 27:6333-45.

18. Kruger S, Mahnken A, Kausch I, Feller AC. Value of clusterin immunoreactivity as a predictive factor in muscle-invasive urothelial bladder carcinoma. Urology 2006; 67:105-9.

19. Gleave ME, Gleave J, Burt HM. The inhibition of angiogenesis by antisense oligonucleotides to clusterin. Angiogenesis 2005; 8:229-8.

20. Bruschi M, Candiano G, Rastaldi MP, Scolari F, Passerini P, Musante L, et al. Depletion of clusterin in renal diseases causing nephrotic syndrome. Kidney Int 2002; 62:2184-94.

21. Hidaka S, Kranzlin B, Gretz N, Witzgall R. Urinary clusterin levels in the rat correlate with the severity of tubular damage and may help to differentiate between glomerular and tubular injuries. Cell Tissue Res 2002; 310:289-6.

22. Saunders JR, Aminian A, McRae JL, O'Farrell KA, Adam WR, Murphy BF. Clusterin depletion enhances immune glomerular injury in the isolated perfused kidney. Kidney Int 1994; 45:817-827.

23. Trougakos IP, Poulakou M, Stathatos M, Chalikia A, Melidonis A, Gonos ES. Serum levels of the senescence biomarker clusterin/apolipoprotein $\mathbf{J}$ increase significantly in diabetes type II and during development of coronary heart disease or at myocardial infarction. Exp Gerontol 2002; 37:1175-1187.

24. Millis AJ, Luciani M, McCue HM, Rosenberg ME, Moulson CL. Clusterin regulates vascular smooth muscle cell nodule formation and migration. J Cell Physiol 2001; 186:210-219.

25. Krijnen PA, Cillessen SA, Manoe R, Muller A, Visser CA, Meijer CJ, et al. Clusterin: a protective mediator for ischemic cardiomyocytes? Am J Physiol Heart Circ Physiol 2005; 289:2193-02.

26. Urbich C, Fritzenwanger M, Zeiher AM, Dimmeler S. Laminar shear stress upregulates the complement-inhibitory protein clusterin: a novel potent defense mechanism against complement-induced endothelial cell activation.Circulation 2000; 101:352-355. 